Original Contribution

\title{
DIFFICULTIES IN THE DIAGNOSIS AND SURGICAL TREATMENT OF XANTOGRANULOMATOUS PYELONEPHRITIS
}

\author{
E. Enchev ${ }^{*}$, G. Minkov², E. Dimitrov², S. Nikolov², A. Petrov ${ }^{3}$, Iv. Ovcharov, \\ Y. Yovtchev ${ }^{2}$ \\ ${ }^{1}$ Department of Clinical Pathology, Medical Faculty, Trakia University, Stara Zagora, Bulgaria \\ ${ }^{2}$ Department of Surgical Diseases and Anestesiology, Medical Faculty, Trakia University, \\ Stara Zagora, Bulgaria \\ ${ }^{3}$ Department of General Surgery, Neurosurgery and Urology, Medical Faculty, Trakia University, \\ Stara Zagora, Bulgaria
}

\begin{abstract}
Purpose: The diagnosis of xanthogranulomatous pyelonephritis is primarily histological. The mandatory studies that have to be performed are: abdominal echography, excretory urography, CT, and renal angiography.

Methods: The current report covers a period of 16 years (1992-2008) and presents clinical material from 9 patients with a long history of nephrolithiasis. In the past, six of them have undergone surgical or extracorporeal lithotripsy.

Results and conclusion: The performed preoperative imaging studies showed kidney tumors, and the operative treatment method used was nephrectomy. The post-operative follow-up did not detect any complications in any of these patients.
\end{abstract}

Key words: surgical management, urinary obstruction, chronic granulomatous process

\section{INTRODUCTION}

For the first time in 1916 Schlagenhaufer describes the xanthogranulomatous pyelonephritis (XGP), and the same was also described in the writings of Putschar and Osterling in the 1930s. Nowadays, XGP is a particular type of inflammatory process affecting renal parenchyma characterized by a disruption of urinary drainage, which is largely the cause of an incorrect diagnosis of neoplasm of the kidney. (1) A majority of cases of XGP have unilateral localization, often associated with urinary tract obstruction, concomitant infection, nephrolithiasis, and diabetes. Some patients have an immunodeficiency disorder. Based on all of this and considering that this is a relatively rare disease, it turns the disease into a diagnostic problem that can put the surgeon in front of a terrible surprise $(2,3)$.

Correspondence to: Emil Enchev, Department of Clinical Pathology, Trakia University, 11 Armeyska Str., Stara Zagora, e-mail:

surgery_umbal_sz@yahoo.com

\section{MATERIALS AND METHODS}

For a period of 16 years 5200 patients were hospitalized and surgically treated in the Department of Urology at University Hospital "Prof. D-r Stoyan Kirkovich", Stara Zagora. During this time, 9 of them $(0.17 \%)$ were diagnosed with unilateral localized XGP. In this group of patients, women occupied $55.55 \%$ (5 women) and males $44.44 \%$ (4 males). The age ranged from 45-65 years (median 55.0). All patients in the past have undergone the following treatment methods: extracorporeal lithotripsy or surgical treatment due to long-standing nephrolithiasis.

The clinical symptoms of patients at hospitalization were heaviness in the lumbar area, pain in one of the lateral canals, general fatigue and subfebrility. Complete blood count test, urine microbiology, abdominal ultrasound scan, CT scan, excretory urography in 4 patients, and renovasography in 2 were performed. 


\section{RESULTS}

Initial laboratory findings showed first degree of anemic syndrome ( $\mathrm{Hb}$ below $120 \mathrm{~g} / \mathrm{l}$ for women and less than $140 \mathrm{~g} / \mathrm{l}$ for men) at 6 (66.66\%) patients and transient leukocytosis up to $14 \times 109$ in $7(77.77 \%)$ patients. Pyuria was diagnosed in $3(33.33 \%)$ patients and transient proteinuria at $5(55.55 \%)$ patients.

Hydronephrosis, deformation of the pyelocalyx system (PCS) and heterogeneous change in parenchyma structure were diagnosed by ultrasound. (Figure 1)

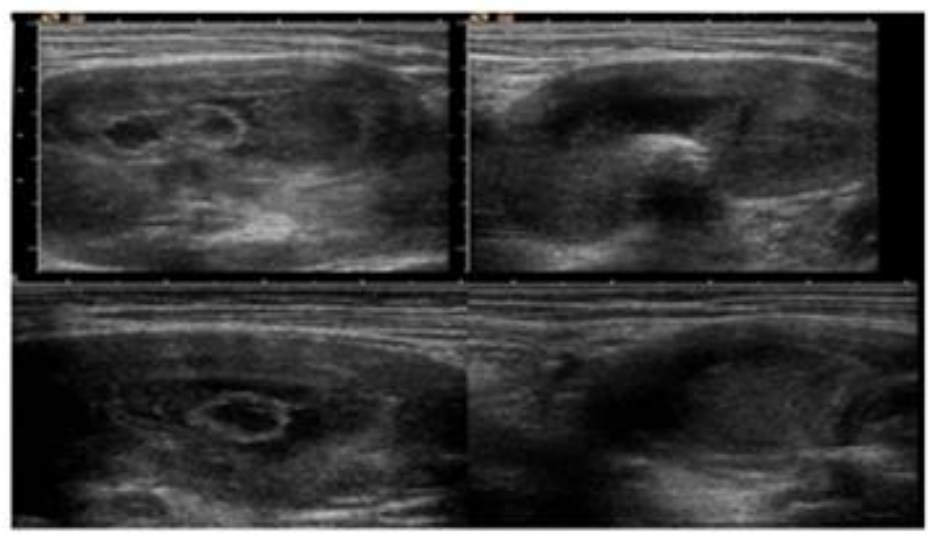

Figure 1. Ultrasonographic structure of parenchyma in a patient with xanthogranulomatous pyelonephritis

The occurrence of pyuria and the subsequent microbiological test resulted in the detection of Escherichia coli in 3 patients $(33.33 \%)$, Proteus mirabilis in 4 patients $(44.44 \%)$ and Staphylococcus aureus in two (22.22\%). So we had the opportunity to trace the effect of antibiotic treatment initiated. This duration included preoperative patient preparation and drug susceptibility to the drug. In six of the patients therapy included cephalosporin first generation, and three others were treated with fluoroquinolones.

In four of the patients, venous urography established an enlarged kidney and a defect in the PCS affected by the inflammatory process. However, there were no visualized and diagnosed disturbances in the secretory and excretory functions. In two patients, renovasography identified chaotic arterial and venous vessels, interpreted as vascular defects typical of a neoplastic process localized in the renal parenchyma. Based on the results of the performed instrumental examinations in the differential diagnosis, we assumed that it was probably a kidney tumor, which was the reason for these patients to be offered surgical treatment.

After written consent, we operated on a scheduled basis. As conventional access, lumbotomy was used in 7 patients, making it extremely difficult for us to continue working with one of these patients. Transabdominal surgical access was undertaken in two patients. Intraoperatively a massive granulomatous tissue emerged from the renal capsule, which invades the lumbar musculature, the spine, the back of the peritoneum and the posterior wall of the colon. (Figure 2)

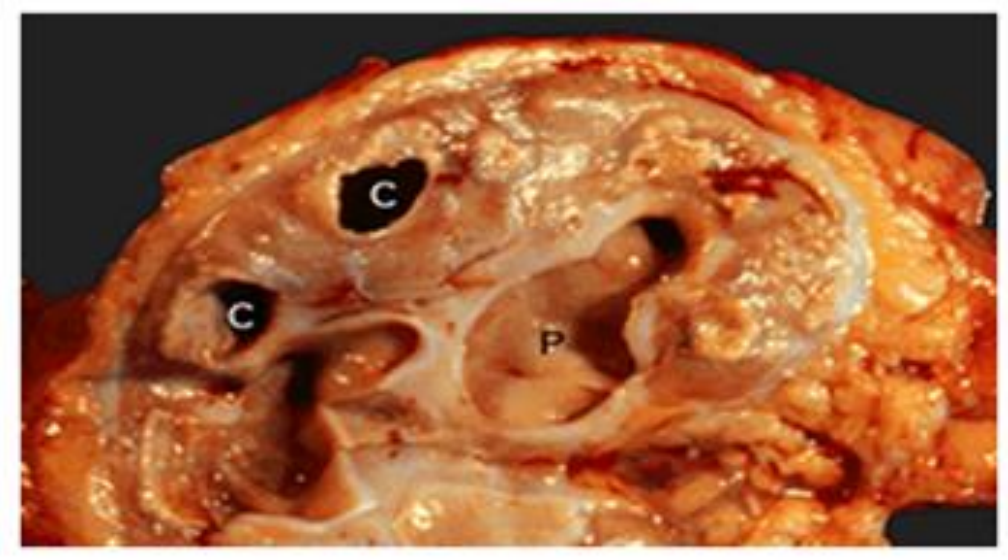

Figure 2. Kidney - longitudinal section, macroscopic view, C-calyx, P-pyelon 
This has greatly hampered further operative work and has led to two operative interventions end with nephrostomy. Nevertheless, we performed an express histological examination of all patients, with the initial results of five of them being a kidney tumor - a hyper-nephroid variant. However, lasting histological results have shown that it is XGP. (Figure 3)

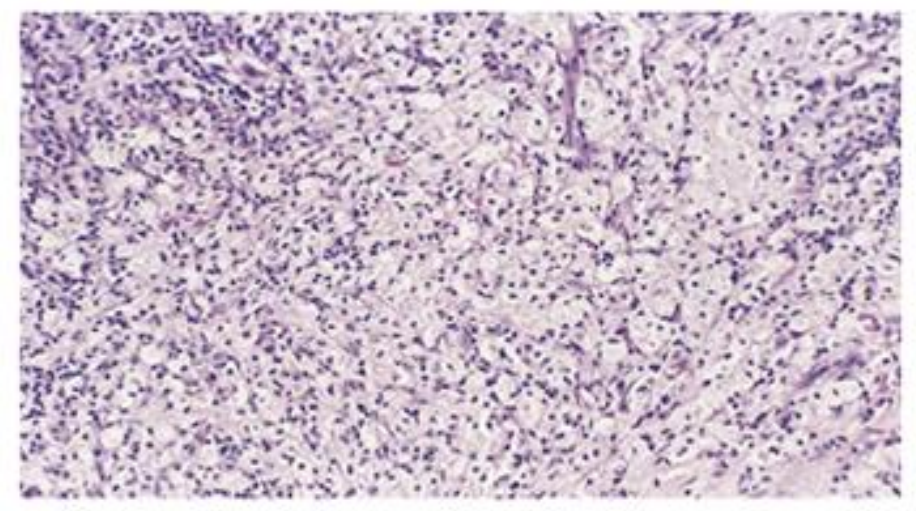

Figure 3. Microscopic structure of renal parenchyma in xanthogranulomatous pyelonephritis - detailed image

\section{DISCUSSION}

As a result of the improved conservative and surgical treatment of the nephrolithiasis, hydronephrotic transformations and pyelonephritis, nowadays the incidence of $\mathrm{XGP}$ is low. Results from our research were no different from the data published in the medical literature (3). The exact etiological cause of the disease is still unknown, but it is generally accepted that the process requires the presence of renal obstruction and concomitant infection. Renal calculosis was found in almost $80 \%$ of patients with XGP. This disease is also often diagnosed in patients with diabetes and/or those with a breakdown in lipid metabolism, which makes them a hypothetical option from an etiological point of view. However, it is more likely that the mechanical obstruction in urine output is a consequence of changes in renal parenchyma rather than a condition for disease progression. This was found in our patients' group where we were able to instrumentally prove that despite the established changes in renal parenchyma there was a lack of disruption in drainage function.

Clinical complaints of patients with this disease remain atypical, making further clinical interpretation of the results difficult. Common symptoms include anorexia, fever, weight loss, and flank pain. Pain in XGP is not colic, but in most cases, it is dull and constant. The urine typically contains leukocytes and bacteria. When urine $\mathrm{pH}$ is alkaline, the microbiological finding in most cases detects Proteus mirabilis, a urease-producing organism. The visualized erythrocytes in the urine are within the normal range or their levels are slightly elevated.

The microbiological diagnosis of this disease helps to some extent control of the concomitant inflammatory process that develops. In our patients, the presence of pyuria was one of the indications of antibiotic therapy targeting a likely causative agent. This therapy preceded the results of the microbiological study and we continued it for several days after surgery. Therapeutic regimens included venous intake of first generation cephalosporin or a representative of fluoroquinolones. In our patients, no replacement of the initial empiric antibiotic therapy was required. Of course, we give two main reasons - the antibiotic policy of the Department of Urology and the judgment of the specialist on the severity and nature of the inflammatory process.

The sequence of the diagnostic methods used prior to surgery did not contribute significantly to the correct diagnosis and the subsequent urgent histological examination caused five patients to have nephrectomy due to renal carcinoma. Even the extended diagnostic algorithm used in our patients did not invalidate the opinion of other authors who also found that $\mathrm{x}$-ray and ultrasound examination did not have the necessary diagnostic value for this disease and could not 
serve as a basis for interpretation of the resulting $x$-ray image $(1,4,6)$.

In support of this, Shah HN et al. (4) described a case of misdiagnosis based on x-ray examination. The same was subsequently the cause of nephrectomy, and the histological examination proves renal tuberculosis and XGP. The instrumental methods thus used, do not have the necessary diagnostic information, sufficient to support the treatment $(1,13)$. Although the disease is a diagnostic problem from the point of view of available instrumental methods, on the other hand, the need for timeliness and adequacy of the surgical intervention is necessary. The question remains: what volume of surgical behavior would be best for the patient with this disease? It is clear that the localization of the xanthogranulomatous process in our patient group covers only one of the two kidneys. This gave us some advantage in treatment tactics, compared to those in the medical literature describing two-sided localization $(5,7)$. In patients with established bilateral disease localization, the partial resection described did not result in the expected outcome but were the cause of a catastrophic outcome (5).

The thoracolumbar incision is preferable to the transabdominal, as it avoids the possibility of further spread of the inflammatory process through the peritoneum. On the other hand, its use in our cases significantly hampered further operative work due to its wide spread. Despite of the difficulties encountered in eight out of nine patients $(88.8 \%)$, the operative method was nephrectomy, although organ-saving methods of surgical treatment were also known. In one patient $(12.2 \%)$ the surgical approach was transabdominal. The nephrectomy as a radical method of treatment in patients with XGP was confirmed in the publications of other authors who believe that partial nephrectomy leads to the appearance of a malfunctioning kidney (5-8). We did not have a technical opportunity to use endoscopic nephrectomy as a method of surgical treatment. In our patients, the inflammatory process also involved other adjacent organs, which significantly hampered the conventional approach. However, this surgical procedure is appropriate and applicable to limited forms of the disease (9-11), although Bercowsky et al. deny its advantage (12).

On the other hand, conventionally performed nephrectomy affected the length of hospital stay, but thanks to it, in $90 \%$ of patients, we achieved complete removal of the kidney with adjacent granulomatous tissue. Remaining part of this tissue, according to some authors, may conceal the presence of a neoplastic process $(13,14)$ or cause the development of postoperative fistula (13). After a year, the screening of patients treated for XGP did not result in the complications described above.

\section{CONCLUSION}

The classic imaging techniques used in urology - ultrasound, intravenous urography, renovasography, and CT most of the time direct the surgeon to the diagnosis of kidney tumor. At the same time, the poor clinical finding from instrumental research and atypical clinical symptoms further complicates diagnosis and directs a differential diagnosis to a likely neoplastic process of the kidney. In our study, we found that urgent histological examination in 5 patients did not result in definitive disease verification. However, the overall prognosis of the disease remains good, and the death from it is relatively rare.

\section{REFERENCES}

1. Hammadah MY, Nicholls CJ, Calder JC, Buick RG, Gornall P, Corkery JJ. Xanthomatous pyelonephritis in childhood: Pre-operatory diagnosis is possible. $\mathrm{Br} J$ Urol. 1994;73:83-86

2. Hitti W, Drachenberg C, Cooper M, Schweitzer E, Cangro C, Klassen D, et al. Xanthogranulomatous pyelonephritis in a renal allograft associated with xanthogranulomatous diverticulitis: report of the first case and review of the literature. Nephrol

Dial Transplant. Nov 2007;22(11):3344-7.

3. Tolkoff-Rubin NE, Cotran RS, Rubin RH. Urinary tract infection, pyelonephritis and reflux nephropathy. Brenner BM editors. The kidney. ed. 6. Philadelphia: W.B. Saunders; 2000

4. Shah HN, Jain P, Chibber PJ. Renal tuberculosis simulating xanthogranulomatous pyelonephritis with contagious hepatic involvement. Int $J$ Urol. Jan 2006;13(1):67-8.

5. Perez LM, Thrasher JB, Anderson EE. Successful management of bilateral xanthogranulomatous pyelonephritis by bilateral partial nephrectomy. $J$ Urol. 1993;149:100-102.

6. Malek RS, Elder JS. Xanthogranulomatous pyelonephritis: a critical analysis of 26 
ENCHEV E., et al.

cases and of the literature. $J$ Urol. May 1978;119(5):589-93.

7. Osca JM, Peiro MJ, Rodrigo M, MartinezJabaloyas JM, Jimenez-Cruz JF. Focal xanthogranulomatous pyelonephritis: partial nephrectomy as definitive treatment. Eur Urol. 1997;32(3):375-9.

8. Nataluk EA, McCullough DL, Scharling EO. Xanthogranulomatous pyelonephritis, the gatekeeper's dilemma: a contemporary look at an old problem. Urology. Mar 1995;45(3):377-80.

9. Borum ML. An unusual case of nephrobronchial and nephrocolonic fistula complicating xanthogranulomatous pyelonephritis. Urology. Sep 1997;50(3):44 3.

10.Borzi PA, Yeung CK. Selective approach for transperitoneal and extraperitoneal endoscopic nephrectomy in children. $J$ Urol. Feb 2004;171(2 Pt 1):814-6; discussion 816.
11.Tunc L, Biri H, Onaran M, Krac M, Yesil S, Bozkirli I. Laparoscopic nephrectomy for xanthogranulomatous pyelonephritis in the absence of kidney stones or clinical urinary infection. Surg Laparosc Endosc Percutan Tech. Dec 2007;17(6):570-2.

12. Bercowsky E, Shalhav AL, Portis A, Elbahnasy AM, McDougall EM, Clayman RV. Is the laparoscopic approach justified in patients with xanthogranulomatous pyelonephritis?. Urology. Sep 1999;54(3):4 37-42; discussion 442-3.

13.Papadopoulos I, Wirth B, Wand H. Xanthogranulomatous pyelonephritis associated with renal cell carcinoma. Report on two cases and review of the literature. Eur Urol. 1990;18(1):74-6.

14.Treadwell TS, Craven DE, Delfin H, Stilmant MM, McCabe WR. Xanthogranulomatous pyelonephritis caused by methicillin-resistant Staphylococcus aureus. Am J Med. 1984;76:533-537. 